\title{
Activated Protein C has No Effect on Pulmonary Capillary Endothelial Function in Septic Patients with Acute Respiratory Distress Syndrome: Association of Endothelial Dysfunction with Mortality
}

\author{
Katerina Kaziani - Alice G. Vassiliou - Anastasia Kotanidou • \\ Chariclea Athanasiou - Ioanna Korovesi - Konstantinos Glynos • \\ Stylianos E. Orfanos
}

Received: October 12, 2017

(C) The Author(s) 2018. This article is an open access publication

\section{ABSTRACT}

Introduction: Pulmonary capillary endothelium-bound (PCEB) angiotensin-converting enzyme (ACE) activity is a direct and quantifiable index of pulmonary endothelial function that decreases early in acute respiratory distress syndrome (ARDS) and correlates with its

Enhanced content To view enhanced content for this article go to https://doi.org/10.6084/m9.figshare.58781 53.

K. Kaziani · A. G. Vassiliou · A. Kotanidou ·

C. Athanasiou · I. Korovesi · K. Glynos ·

S. E. Orfanos

First Department of Critical Care Medicine and Pulmonary Services, GP Livanos and M Simou Laboratories, Evangelismos Hospital, Medical School of the National and Kapodistrian University of Athens, Athens, Greece

\section{A. Kotanidou}

First Department of Critical Care Medicine and Pulmonary Services, Evangelismos Hospital, Medical School of the National and Kapodistrian University of Athens, Athens, Greece

\section{S. E. Orfanos}

Second Department of Critical Care, Attikon Hospital, Medical School of the National and Kapodistrian University of Athens, Haïdari, Athens, Greece

\section{K. Kaziani $(\bowtie)$}

5th Department of Internal Medicine and Infectious Diseases Unit, Evangelismos Hospital, Athens, Greece

e-mail: katkaziani@hotmail.com severity. Endothelial dysfunction is a major pathophysiology that underlies sepsis-related ARDS. Recombinant human activated protein C (rhAPC), now withdrawn from the market, has been used in the recent past as an endothelialprotective treatment in patients with septic organ dysfunction.

Methods: We investigated the effect of rhAPC on pulmonary endothelial function in 19 septic patients suffering from ARDS. Applying indicator-dilution type techniques, we measured single-pass transpulmonary percent metabolism $(\% M)$ and hydrolysis (v) of the synthetic, biologically inactive, and highly specific for ACE substrate, ${ }^{3}$ H-benzoyl-Phe-Ala-Pro (BPAP), under first-order reaction conditions, and calculated lung functional capillary surface area before and after treatment with rhAPC.

Results: Pulmonary endothelium ACE activity was severely impaired in septic patients with ARDS, and was not affected by rhAPC treatment. Additionally, poor outcome was related to a more profound decrease in PCEB-ACE activity. Angiotensin-converting enzyme-substrate utilization was statistically significantly lower in non-survivors as compared to survivors, with no changes over time within each group: BPAP \%M: $32.7 \pm 3.4 \%$ at baseline to $25.6 \pm 2.9 \%$ at day 7 in survivors versus $20.8 \pm 2.8$ to $15.5 \pm 5 \%$, respectively, in nonsurvivors $(p=0.044)$, while hydrolysis $(v)$ : $0.41 \pm 0.06$ at baseline to $0.30 \pm 0.04$ at day 7 in survivors compared to $0.24 \pm 0.04$ to 
$0.18 \pm 0.06$, respectively, in non-survivors $(p=0.049)$.

Conclusion: rhAPC administration in septic patients with ARDS did not improve PCEB-ACE activity indices. However, these indices might be useful in the early recognition of septic patients with ARDS at high risk of mortality.

Keywords: ACE; ARDS; Prognosis; rhAPC; Sepsis

\section{INTRODUCTION}

The pulmonary microvascular endothelium possesses numerous metabolic properties, many of which are catalyzed by ectoenzymes expressed on the luminal pulmonary endothelial surface [1]. Angiotensin-converting enzyme (ACE) is the ectoenzyme that catalyzes the conversion of angiotensin I to angiotensin II, as well as the degradation of bradykinin, thus regulating the vascular tone. Given the strategic location of the lungs, and the extent of the pulmonary capillary endothelial surface, the pulmonary vasculature is the main site of ACE activity [1].

Pulmonary capillary endothelium-bound (PCEB) ACE activity has been widely studied in animals and humans by indicator-dilution techniques and has been shown to provide a quantifiable, direct and sensitive measurement of endothelial function under normal conditions and pulmonary diseases $[1,2]$.

In acute respiratory distress syndrome (ARDS), most commonly occurring during sepsis, pulmonary capillary endothelial cells appear to be among the first lung cells to be insulted, leading to impaired metabolic functionality $[3,4]$. In this respect, PCEB-ACE activity has been shown to decrease early during ARDS and to correlate with its severity [5].

Activated protein C (APC) system dysregulation plays an important role in the pathogenesis of sepsis-associated organ dysfunction [6]. In 2004, the use of recombinant human APC (rhAPC; drotrecogin alfa (activated); Xigris; Eli Lilly, Indianapolis, IN, USA) in patients with sepsis-induced organ failure was recommended based on the results of the PROWESS study
$[7,8]$. We hypothesized that rhAPC administration in septic patients with ARDS would improve pulmonary endothelial function, as assessed by PCEB-ACE activity indices.

It should be noted that the drug was withdrawn from the market after the negative results of the PROWESS-SHOCK study [9]. However, our study on the effect of rhAPC on pulmonary endothelial function of septic patients with ARDS was performed during the period that the drug was in use in patients with sepsis-induced organ dysfunction.

\section{METHODS}

\section{Compliance with Ethics Guidelines}

The study was approved by the Evangelismos Hospital Research Ethics Committee (94/9-92003). All procedures followed were in accordance with the ethical standards of the responsible committee on human experimentation (institutional and national) and with the Helsinki Declaration of 1964, also as revised in 2013. Informed written consent was obtained from all patients' next-of-kin prior to any study procedure. Our study was conducted and completed in 2004, before the International Committee of Medical Journal Editors initiated the policy requiring investigators to deposit information about trial designs. Consequently, our study was not registered.

\section{Study Population and Measurements}

In 2004, 19 critically-ill septic patients with ARDS treated with rhAPC were enrolled in the study. All subjects were hospitalized in a general intensive care unit (ICU), were mechanically ventilated, and had at least two organ dysfunctions consequent to infection, one of which was sepsis-associated respiratory dysfunction. All patients suffered from severe sepsis or septic shock and, furthermore, all patients had ARDS except three who had acute lung injury, according to the respective definitions at the time of the study $[10,11]$; based on the 
current definitions, they are all considered to have suffered from sepsis or septic shock and ARDS $[12,13]$. The decision to treat with rhAPC was taken by the patients' attending physicians, in compliance with the administration criteria valid at that time [8]. They all received rhAPC at $24 \mu \mathrm{g} / \mathrm{kg} / \mathrm{h}$ for $96 \mathrm{~h}$ in a continuous IV drip. All the precautions were taken regarding the risk of bleeding before and after major or minor surgical procedures. Major bleeding events were not noticed. After the completion of the study, patients were followed up and complete clinical and laboratory data were recorded for 28 days or until their discharge from the ICU. At the end of the study, the subjects were grouped according to their outcome ( 28 days survival or prior discharge from the ICU) into survivors and nonsurvivors.

PCEB-ACE activity was measured prior to initiation of rhAPC treatment (measurement \#1 at baseline), during the 96-h infusion (measurement \#2 at $24 \mathrm{~h}$; measurement \#3 at $48 \mathrm{~h}$; measurement \#4 at $96 \mathrm{~h}$ ) and 3 days after the end of the rhAPC infusion (measurement \#5, day 7). No patient was under ACE inhibitors. Each patient's baseline measurement served as control of him/herself.

A detailed description of the methodology used is given elsewhere [2]. Trace amounts of the radiolabelled synthetic ACE substrate ${ }^{3}[\mathrm{H}]$ benzoyl-Phe-Ala-Pro (BPAP) were injected as a rapid bolus into a (jugular or subclavian) central vein, and arterial blood was withdrawn into a fraction collector for analysis.

Single transpulmonary pass substrate utilization is expressed as percent metabolism $(\% M)$ and transpulmonary hydrolysis $\left(v=[E] \times t_{\mathrm{c}} \times k_{\text {cat }} / K_{\mathrm{m}}\right)$, where $[E]$ equals the enzyme concentration available for reaction, $t_{\mathrm{c}}$ the capillary transit time, $k_{\text {cat }}$ the catalytic rate constant, and $K_{\mathrm{m}}$ the Michaelis-Menten constant. Functional capillary surface area (FCSA) was additionally calculated as hydrolysis $\times$ pulmonary plasma flow and depends on the total enzyme mass that is available for reaction, and the constants $k_{\text {cat }}$ and $K_{\mathrm{m}}$. Both $v$ and $\% M$ are indices that reflect ACE activity per capillary, while FCSA is a reflection of ACE activity per vascular bed $[1,2,14]$.

\section{Statistical Analysis}

Data are presented as mean $\pm \mathrm{SE}$, or median (interquartile range) when data distribution was skewed. Comparisons between groups were made by Student's $t$ test or Mann-Whitney test, when appropriate, for continuous data, and Chi square test for qualitative data. Differences within time were examined by the Friedman test. Mixed effect models were fitted to examine the effect of time, outcome and their interaction. The Tukey-Kramer test was used for multiple comparisons adjustment. All $p$ values are two-sided. Differences were considered significant at $p<0.05$.

\section{RESULTS}

\section{Characteristics of the Study Population}

Patient baseline demographic and clinical data are shown in Table 1. Patients were grouped according to their clinical outcome (28 days survival) as survivors $(n=9)$ and non-survivors $(n=10)$. There were no statistically significant differences between the two groups in baseline characteristics.

\section{The Effect of Recombinant Human APC on Pulmonary Endothelial ACE Activity and FCSA}

In all patients, BPAP $\% M$ did not significantly change during the study period: from $26.4 \pm 2.6 \%$ (baseline) to $24.4 \pm 2.9 \%$ at the end of the 96-h rhAPC infusion, and to $22.5 \pm 2.8$ at the end of the 7 days study (Fig. $1 \mathrm{a} ; p=0.541$ ). In a similar pattern a non-significant fluctuation of hydrolysis $(v)$ was observed in all patients from $0.32 \pm 0.04$ at baseline to $0.29 \pm 0.04$ at day 4 and to $0.27 \pm 0.04$ at day 7 (Fig. 1b; $p=0.641$ ). FCSA at baseline was $1550 \pm 212 \mathrm{ml} / \mathrm{min}$, while similar values were obtained at day $4(1549 \pm 276 \mathrm{ml} / \mathrm{min})$ and at day $7(1561 \pm 292 \mathrm{ml} / \mathrm{min})($ Fig. $1 \mathrm{c} ; p=0.652)$. 
Table 1 Demographic and clinical data of the 19 patients enrolled in the study

\begin{tabular}{lllll}
\hline & All patients (19) & Survivors (9) & Non-survivors (10) & P value \\
\hline Age (years), mean \pm SE & $64 \pm 3.3$ & $61.8 \pm 4.9$ & $66.3 \pm 4.5$ & 0.503 \\
Male sex, $n$ (\%) & $15(79)$ & $7(78)$ & $8(80)$ & 0.906 \\
Days in ICU, median (IQR) & $4(2-8)$ & $3(1.5-6)$ & $6(2.75-17)$ & 0.09 \\
Days on MV, median (IQR) & $4(2-9)$ & $3(1.5-6)$ & $6(2.75-11.75)$ & 0.119 \\
Cause of ARDS, $n$ (\%) & & & & 0.809 \\
Direct & $9(47)$ & $4(44)$ & $5(50)$ & \\
Indirect & $10(53)$ & $5(66)$ & $5(50)$ & \\
\hline
\end{tabular}

Data are expressed as number of patients $(n)$, mean \pm SE, or median (25-75\% interquartile range)

$A R D S$ acute respiratory distress syndrome, ICU intensive care unit, $I Q R$ interquartile range, $M V$ mechanical ventilation

\section{PCEB-ACE Activity and Outcome (28 Days Survival)}

Neither \%M nor hydrolysis changed significantly with time in either group. In survivors, BPAP \%M tended to decrease from $32.7 \pm 3.4 \%$ at baseline to $25.6 \pm 2.9 \%$ at day 7 , and in nonsurvivors from $20.8 \pm 2.8 \%$ at baseline to $15.5 \pm 5 \%$ at day 7. Likewise, $v$ tended to decrease from $0.41 \pm 0.06$ at baseline to $0.30 \pm 0.04$ at day 7 in survivors, and $0.24 \pm 0.04$ to $0.18 \pm 0.06$ in non-survivors (Fig. 2a, b; $p=0.489$ and $p=0.499$, respectively). Nonetheless, ACE substrate utilization was significantly lower in non-survivors as compared to survivors (Fig. 2a, b; $p=0.044$ and $p=0.049)$.

\section{Functional Capillary Surface Area and Outcome (28-day Survival)}

FCSA increased slightly, from $1760 \pm 327 \mathrm{ml} /$ $\mathrm{min}$ at baseline to $1890 \pm 338 \mathrm{ml} / \mathrm{min}$ at day 7 in survivors, while in the non-survivors FCSA dropped from $1362 \pm 276$ to $821 \pm 402 \mathrm{ml} / \mathrm{min}$. It tended to be higher in survivors, without, however, reaching significance (Fig. 2c, $p=0.075$ ). It should be noted that the obtained values might be influenced by differences in cardiac output per patient, observed during the study (data not shown), since they affect FCSA measurements (see "Methods").

\section{Disease Severity and Lung Injury Scores}

Patients' $\mathrm{PO}_{2} / \mathrm{FiO}_{2}$ did not significantly change with time (Fig. 3a; $p=0.458$ ). However, when patients were divided into survivors and nonsurvivors, the former revealed significantly higher values than the latter (Fig. $3 b ; p=0.032$ ). Similar patterns were observed for lung injury scores (LIS) (Fig. 3c; $p=0.726$ ). Again, survivors had significantly lower LIS than non-survivors (Fig. 3d; $p=0.025$ ). SOFA scores decreased with time in all patients (Fig. $4 \mathrm{a} ; p=0.007$ ). The same pattern (i.e. decrease with time) was observed in both survivors and non-survivors, and were lower in survivors than in non-survivors (Fig. $4 \mathrm{~b} ; p=0.001$ and $p=0.04$, respectively). Patients' APACHE II scores also decreased with time in all patients (Fig. 4c; $p=0.033$ ). The same pattern (i.e. decrease with time) was observed in both survivors and nonsurvivors (Fig. $4 \mathrm{~d} ; p=0.045$ ). However no differences were noted between the two groups (Fig. 4d; $p=0.189$ ).

It is worth mentioning that PCEB-ACE activity indices were the only significantly different parameters between survivors and nonsurvivors at baseline; no other disease or lung injury severity score was significantly different at baseline (data not shown). More specifically, in survivors, BPAP $\% M$ at baseline was $32.7 \pm 3.4 \%$ compared to $20.8 \pm 2.8 \%$ in nonsurvivors (Mann-Whitney, $p=0.017$ ). Baseline 

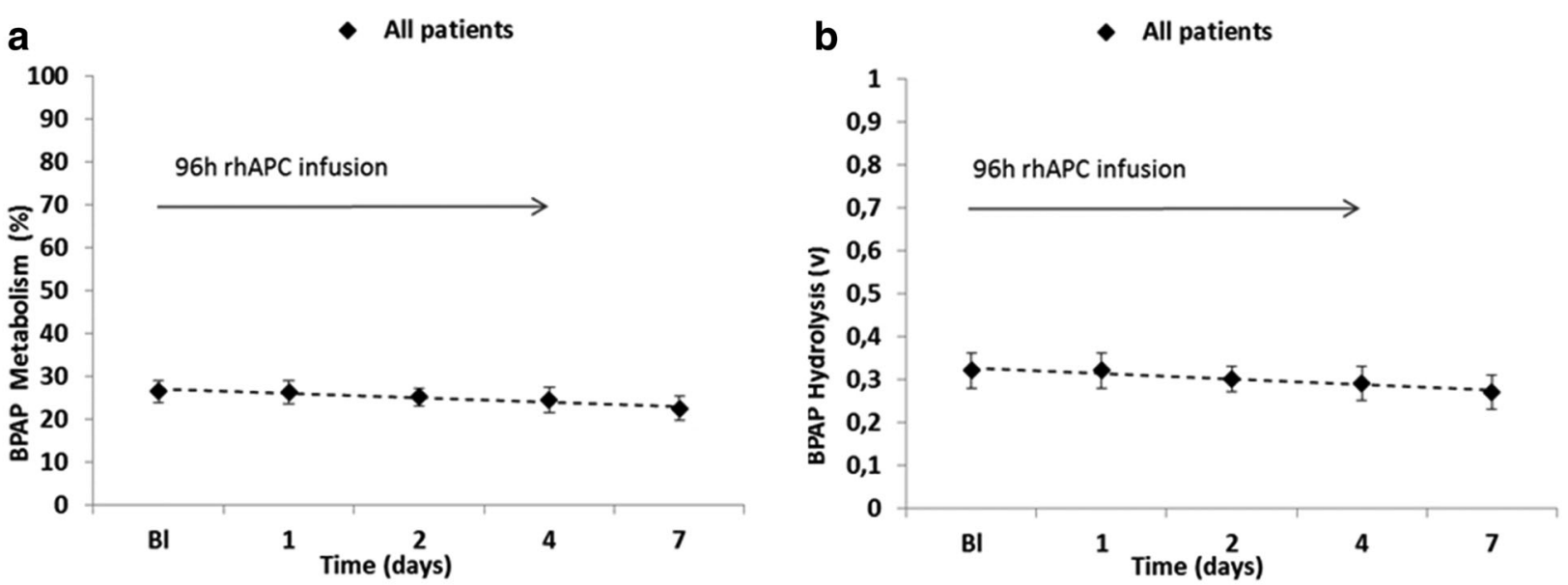

- All patients

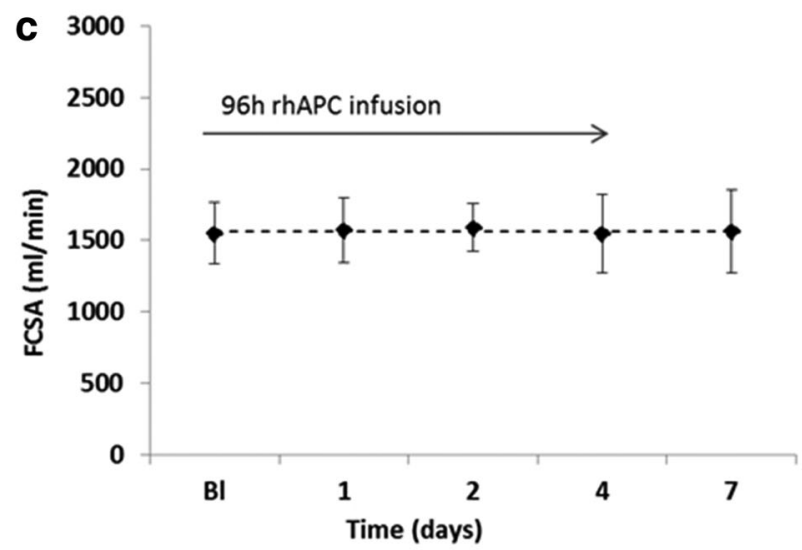

Fig. 1 The effect of recombinant human APC on pulmonary endothelial ACE activity and FCSA. PCEB-ACE activity was measured prior to initiation of treatment with rhAPC (measurement \#1 at baseline, $B l$ ), during the $96 \mathrm{~h}$ infusion (measurement \#2 at $24 \mathrm{~h}$, measurement \#3 at $48 \mathrm{~h}$, measurement $\# 4$ at $96 \mathrm{~h}$ ) and 3 days after the end of the rhAPC infusion (measurement \#5 at day 7). Substrate (BPAP) percent metabolism $(\% M)(\mathbf{a})$, BPAP hydrolysis

hydrolysis $(v)$ was $0.41 \pm 0.06$ in survivors and $0.24 \pm 0.04$ in non-survivors (Mann-Whitney, $p=0.017)$.

\section{DISCUSSION}

In sepsis, the dysregulated interaction between inflammation and coagulation underlies organ dysfunction [6]. On this basis, it was suggested that natural anticoagulants, that are down- (v) (b) and functional capillary surface area (FCSA) (c) were estimated in all patients over time. Data are presented as mean $\pm \mathrm{SE}$. The Friedman test was used, $p>0.05 . B P A P{ }^{3}[\mathrm{H}]$ benzoyl-Phe-Ala-Pro, FCSA functional capillary surface area, $P C E B-A C E$ pulmonary capillary endothelium-bound angiotensin converting enzyme, $r h A P C$ recombinant human activated protein $\mathrm{C}$

regulated in sepsis, could be used as a salvage therapy in septic organ failure. Recombinant human APC was the only anticoagulant initially shown to be effective in decreasing mortality in severely septic patients [7]. Furthermore, several experimental studies have documented the beneficial effects of the treatment with rhAPC (intravenous or inhaled) on inflammation-induced lung injury [15-19].

Pulmonary capillary ACE activity has been proved to be a reliable index of endothelial 


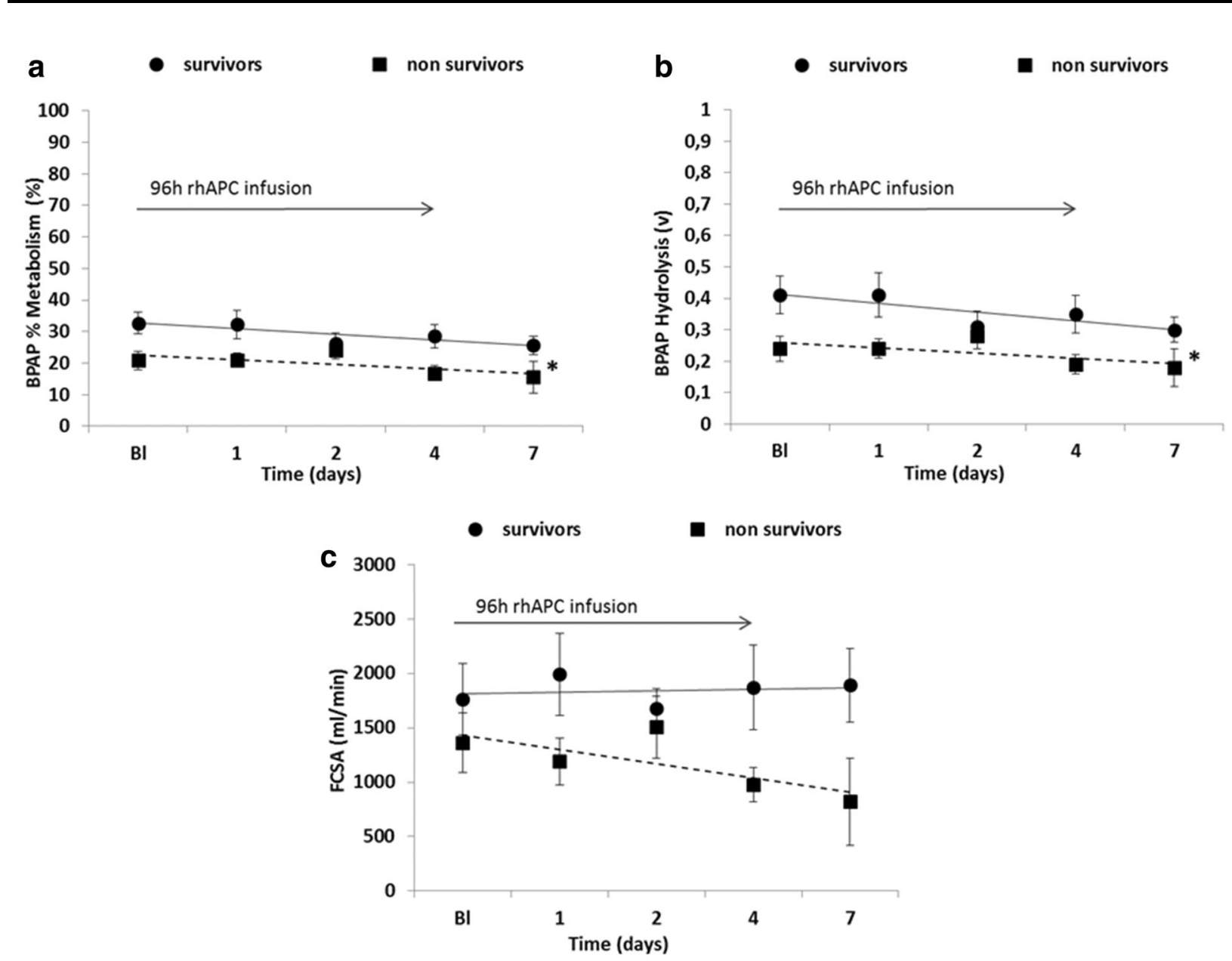

Fig. 2 PCEB-ACE activity and outcome (28-day survival). PCEB-ACE activity was measured prior to initiation of treatment with rhAPC (measurement $\# 1$ at baseline, $B l$ ), during the 96-h infusion (measurement \#2 at $24 \mathrm{~h}$, measurement \#3 at $48 \mathrm{~h}$, measurement $\# 4$ at $96 \mathrm{~h}$ ) and 3 days after the end of the rhAPC infusion (measurement \#5 at day 7). Patients were grouped according to their clinical outcome (28-day survival) as survivors $(n=9)$ and non-survivors $(n=10)$. Substrate (BPAP) percent

dysfunction in variable pulmonary diseases $[1,5,20]$. Contrary to other surrogate markers of endothelial injury, such as circulating adhesion molecules and other soluble endothelialderived proteins, its measurement by means of indicator-dilution type techniques provides a direct and sensitive quantification of pulmonary endothelial function at the patients' bedside. Both substrate transpulmonary hydrolysis ( $v$ ) and FCSA have been shown to metabolism $(\% M)(\mathbf{a})$, BPAP hydrolysis $(v)(\mathbf{b})$ and functional capillary surface area (FCSA) (c) were estimated in all patients with time. Data are presented as mean \pm SE. The mixed effect model was used, ${ }^{*} p<0.05$. BPAP ${ }^{3}[\mathrm{H}]$ benzoyl-Phe-Ala-Pro, FCSA functional capillary surface area, $P C E B-A C E$ pulmonary capillary endothelium-bound angiotensin converting enzyme, $r h A P C$ recombinant human activated protein $\mathrm{C}$

decrease early during the ARDS continuum and to be inversely related to LIS [5].

In this study we evaluated baseline PCEBACE activity in a homogenous, septic population with ARDS, in the sense that they all fulfilled the criteria for rhAPC administration. We rationalized that a beneficial effect of rhAPC on pulmonary microcirculation would be depicted as an amelioration of the PCEB-ACE activity indices. Our hypothesis was not confirmed: 

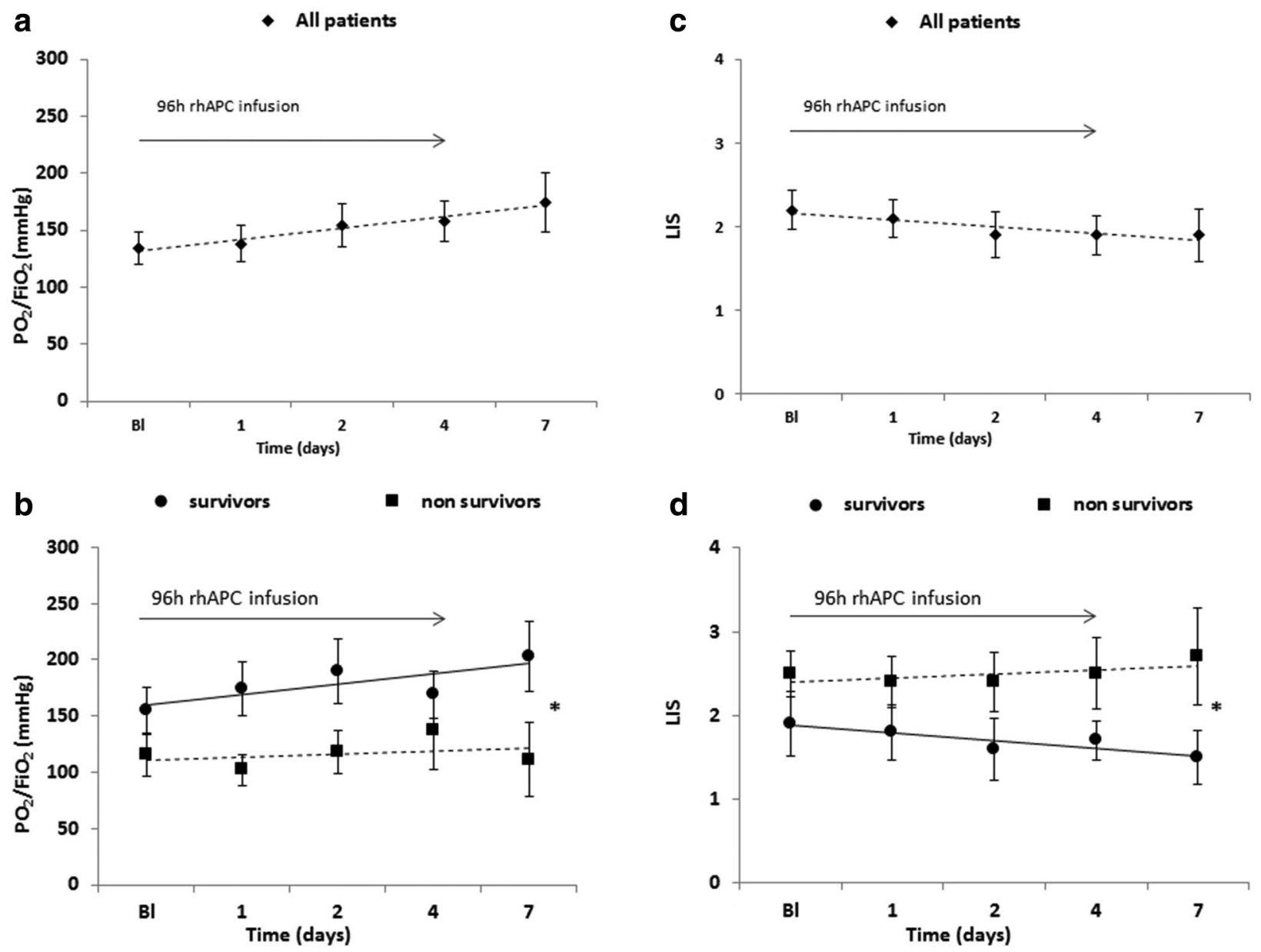

Fig. 3 Lung disease severity scores $\left(\mathrm{PO}_{2} / \mathrm{FiO}_{2}\right.$ and LIS). $\mathrm{PO}_{2} / \mathrm{FiO}_{2}$ was measured prior to initiation of treatment with rhAPC (measurement \#1 at baseline, $B l$ ), during the 96-h infusion (measurement \#2 at $24 \mathrm{~h}$, measurement \#3 at $48 \mathrm{~h}$, measurement $\# 4$ at $96 \mathrm{~h}$ ) and 3 days after the end of the rhAPC infusion (measurement \#5 at day 7). Results are given for the whole cohort (a) and for survivors versus non-survivors (b). Lung injury scores $(L I S)$ were measured prior to initiation of treatment with rhAPC (measurement

rhAPC treatment had no significant effect on pulmonary endothelial function as estimated by this method. Our results coincided with the uncertainty that emerged about the efficacy and the concerns regarding the use of rhAPC in the treatment of sepsis. The PROWESS-SHOCK study that was conducted for the re-examination of the risk/benefit profile of rhAPC failed to document a mortality benefit [9], leading to the withdrawal of the product from the market. Despite rhAPC withdrawal, related research has

$\# 1$ at baseline, $B l$ ), during the 96 -h infusion (measurement $\# 2$ at $24 \mathrm{~h}$, measurement \#3 at $48 \mathrm{~h}$, measurement \#4 at $96 \mathrm{~h}$ ) and 3 days after the end of the rhAPC infusion (measurement \#5 at day 7). Results are given for the whole cohort (c) and for survivors versus non-survivors (d). Data are presented as mean \pm SE. a, c The Friedman test was used, $p>0.05$. b, d The mixed effect model was used, ${ }^{*} p<0.05$. $r h A P C$ recombinant human activated protein $\mathrm{C}$

been recently published, driving us to present these previously obtained data. In this context, a study using the pulmonary leak index of 67Gallium transferrin technique to measure alveolo-capillary permeability in patients with ARDS treated with rhAPC also failed to reveal a beneficial effect of the treatment [21].

In our study population, the estimated BPAP percent metabolism and hydrolysis were considerably decreased compared to the values of normal volunteers with no lung pathologies 

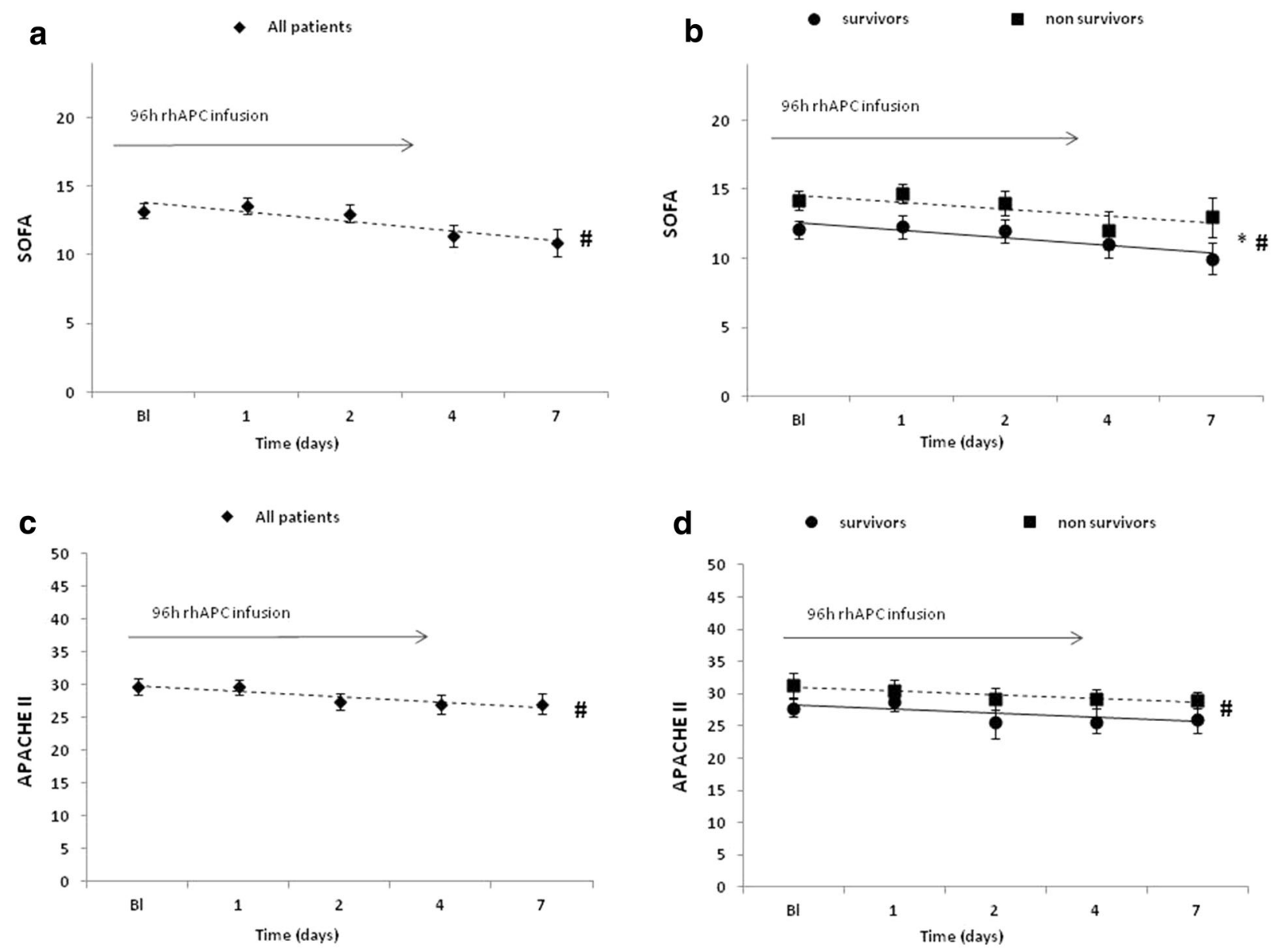

Fig. 4 Disease severity scores. SOFA scores were measured in all patients prior to initiation of treatment with rhAPC (measurement \#1 at baseline, $B l$ ), during the 96 -h infusion (measurement $\# 2$ at $24 \mathrm{~h}$, measurement $\# 3$ at $48 \mathrm{~h}$, measurement \#4 at $96 \mathrm{~h}$ ) and 3 days after the end of the rhAPC infusion (measurement \#5 at day 7). Results are given for the whole cohort (a) and for survivors versus non-survivors (b). APACHE II scores were measured in all patients prior to initiation of treatment with rhAPC (measurement \#1 at baseline, $B l$ ), during the 96 -h infusion (measurement $\# 2$ at $24 \mathrm{~h}$, measurement $\# 3$ at $48 \mathrm{~h}$,

$[1,2,22]$. Further analysis of our results attempted to examine a possible relation of PCEB-ACE activity with patient outcome. Indeed, poor outcome (defined as mortality) was associated with more prominent pulmonary capillary endothelial dysfunction, as expressed by the significantly lower values of PCEB-ACE activity indices (i.e. $\% M$ and $v$ ). These results were in accordance with the

measurement \#4 at $96 \mathrm{~h}$ ) and 3 days after the end of the rhAPC infusion (measurement \#5 at day 7). Results are given for the whole cohort (c) and for survivors versus non-survivors (d). Data are presented as mean \pm SE. a, c The Friedman test was used, ${ }^{\#} p<0.05$ with time. b, d The mixed effect model was used, ${ }^{\#} p<0.05$ with time and ${ }^{*} p<0.05$ between groups. APACHE Acute physiology and chronic health evaluation, $r h A P C$ recombinant human activated protein $\mathrm{C}, S O F A$ sequential organ failure assessment

estimations of disease and respiratory severity indices and scores: when mixed effect models were fitted, non-survivors were found to express worse $\mathrm{PO}_{2} / \mathrm{FiO}_{2}$, as well as LIS and SOFA scores. However, it is worth mentioning that the values of \%M and hydrolysis $(v)$ were statistically lower in the non-survivors group from the very first measurement (baseline measurement) compared to survivors, in contrast to all other 
measured disease severity indices, which showed no significant difference between the two groups at baseline (data not shown). This may denote a superiority of the PCEB-ACE activity indices in providing early information on outcome.

The complex pathophysiology of sepsis and the heterogeneity of septic patients are perhaps reasons why clinicians are still lacking specific treatment options for this syndrome. Our study population consisted of septic patients with ARDS and similar clinical severity, with the limitation of the small size. Nevertheless, our results support a potential implementation of direct markers of endothelial dysfunction in everyday clinical practice. Such markers accompanied by clinical severity scores and other surrogate indices might serve the need for better risk stratification and personalized treatment in sepsis.

\section{CONCLUSION}

In our cohort of septic patients with ARDS, pulmonary endothelium ACE activity was severely impaired, denoting pulmonary endothelial dysfunction. Our results showed that rhAPC treatment had no effect on pulmonary endothelium function. However, we were able to demonstrate that PCEB-ACE activity indices quantitatively expressed the endothelial pathophysiology that underlies sepsis-associated ARDS. Furthermore, they seem to represent early and possibly more sensitive markers of outcome compared to other wellestablished clinical severity indices and scores. It should be noted that the indicator-dilution techniques are arduous procedures, not easily performed in the everyday clinical practice. Future studies using "less demanding" techniques at the bedside are needed in an effort to develop clinically useful tests [23].

\section{ACKNOWLEDGEMENTS}

Funding. This work was funded by an unrestricted grant by Pharmaserve-Lilly, and by the nonprofit institute "THORAX" Research Center for Intensive and Emergency Thoracic Medicine, Athens, Greece. This article is based on work from COST Action "Raman-based applications for clinical diagnostics-Raman4Clinics" (BM 1401), supported by COST (European Cooperation in Science and Technology). No funding or sponsorship was received for the publication of the article.

Medical Writing, Editorial, and Other Assistance. The authors would like to thank the nursing staff of the Evangelismos Hospital ICU for their assistance.

Authorship. All named authors meet the International Committee of Medical Journal Editors (ICMJE) criteria for authorship for this manuscript, take responsibility for the integrity of the work as a whole, and have given final approval to the version to be published.

Prior Presentation. This manuscript has been previously presented as an oral presentation at the Conference of the Hellenic Sepsis Study Group; Critically Ill Patients: From Translational Research to Clinical Management, June 17-18, 2017, Kalamata, Greece.

Disclosures. At the time of the study, Katerina Kaziani' s affiliation was First Department of Critical Care Medicine and Pulmonary Services, GP Livanos and M Simou Laboratories, Medical School of the National and Kapodistrian University of Athens, Evangelismos Hospital, Athens, Greece. Her current affiliation is 5th Department of Internal Medicine and Infectious Diseases Unit, General Hospital "Evangelismos", Athens, Greece. Stylianos E. Orfanos has received an unrestricted grant and speaker honoraria from Pharmaserve-Lilly. Katerina Kaziani, Alice G. Vassiliou, Anastasia Kotanidou, Chariclea Athanasiou, Ioanna Korovesi, and Konstantinos Glynos have nothing to disclose.

Compliance with Ethics Guidelines. The study was approved by the Evangelismos Hospital Research Ethics Committee (94/9-92003). All procedures followed were in 
accordance with the ethical standards of the responsible committee on human experimentation (institutional and national) and with the Helsinki Declaration of 1964, as revised in 2013. Informed written consent was obtained from all patients' next-of-kin prior to any study procedure.

Data Availability. The datasets generated during and/or analyzed during the current study are available from the corresponding author on reasonable request.

Open Access. This article is distributed under the terms of the Creative Commons Attribution-NonCommercial 4.0 International License (http://creativecommons.org/licenses/ by-nc/4.0/), which permits any noncommercial use, distribution, and reproduction in any medium, provided you give appropriate credit to the original author(s) and the source, provide a link to the Creative Commons license, and indicate if changes were made.

\section{REFERENCES}

1. Orfanos SE, Langleben D. Metabolic and clearance function at the pulmonary microvascurar endothelial surface in pulmonary hypertension. In: Yuan JXJ, et al., editors. Textbook of pulmonary vascular disease. New York: Springer; 2011. p. 105-15.

2. Orfanos SE, Langleben D, Khoury J, et al. Pulmonary capillary endothelium-bound angiotensinconverting enzyme activity in humans. Circulation. 1999;99:1593-9.

3. Millar FR, Summers C, Griffiths MJ, Toshner MR, et al. The pulmonary endothelium in acute respiratory distress syndrome: insights and therapeutic opportunities. Thorax. 2016;71:462-73.

4. Ware LB, Matthay MA. The acute respiratory distress syndrome. $\mathrm{N}$ Engl J Med. 2000;342(18):1334-49.

5. Orfanos SE, Armaganidis A, Glynos C, et al. Pulmonary capillary endothelium-bound ACE activity in acute lung injury. Circulation. 2000;102:2011-8.

6. Levi M, van der Poll T, Schultz M. Systemic versus localized coagulation activation contributing to organ failure in critically ill patients. Semin Immunopathol. 2012;34:167-79.

7. Bernard GR, Vincent JL, Laterre PF, Recombinant human protein $C$ Worldwide Evaluation in Severe Sepsis (PROWESS) Study Group, et al. Efficacy and safety of recombinant human activated protein $\mathrm{C}$ for severe sepsis. N Eng J Med. 2001;344:699-709.

8. Dellinger RP, Carlet JM, Masur H, et al. Surviving Sepsis Campaign guidelines for management of severe sepsis and septic shock. Surviving Sepsis Campaign Management Guidelines Committee. Crit Care Med. 2004;32:858-73.

9. Ranieri VM, Thompson BT, Barie PS, PROWESSSHOCK Study Group, et al. Drotrecogin alfa (activated) in adults with septic shock. N Engl J Med. 2012;366:2055-64.

10. American College of Chest Physicians/Society of Critical Care Medicine Consensus Conference. Definitions for sepsis and organ failure and guidelines for the use of innovative therapies in sepsis. Crit Care Med. 1992;20:864-74.

11. Bernard GR, Artigas A, Brigham KL, Carlet J, Falke K, Hudson L, Lamy M, Legall JR, Morris A, Spragg R, The American-European Consensus Conference on ARDS. Definitions, mechanisms, relevant outcomes, and clinical trial coordination. Am J Respir Crit Care Med. 1994;149:818-24.

12. Singer M, Deutschman CS, Seymour CW, et al. The third international consensus definitions for sepsis and septic shock (Sepsis-3). JAMA. 2016;315:801-10.

13. ARDS Definition Task Force, Ranieri VM, Rubenfeld GD, Thompson BT, Ferguson ND, Caldwell E, Fan E, Camporota L, Slutsky AS. Acute respiratory distress syndrome: the Berlin definition. JAMA. 2012;20(307):2526-33.

14. Catravas JD, White RE. Kinetics of pulmonary angiotensin-converting enzyme and 5'-nucleotidase in vivo. J Appl Physiol. 1984;57:1173-81.

15. Murakami K, Okajima K, Uchiba M, et al. Activated protein C prevents LPS-induced pulmonary vascular injury by inhibiting cytokine production. Am J Phys. 1997;272:197-202.

16. Nick JA, Coldren CD, Geraci MW, et al. Recombinant human activated protein $C$ reduces human endotoxin-induced pulmonary inflammation via inhibition of neutrophil chemotaxis. Blood. 2004;104:3878-85.

17. Kotanidou A, Loutrari H, Papadomichelakis E, et al. Inhaled activated protein $\mathrm{C}$ attenuates lung injury induced by aerosolized endotoxin in mice. Vascul Pharmacol. 2006;45:134-40. 
18. Slofstra SH, Groot AP, Maris NA, Reitsma PH, Cate HT, Spek CA. Inhalation of activated protein $C$ inhibits endotoxin-induced pulmonary inflammation in mice independent of neutrophil recruitment. Br J Pharmacol. 2006;149:740-6.

19. Maniatis NA, Letsiou E, Orfanos SE, et al. Inhaled activated protein $\mathrm{C}$ protects mice from ventilatorinduced lung injury. Crit Care. 2010;14:R70.

20. Glynos C, Athanasiou C, Kotanidou A, et al. Preclinical pulmonary capillary endothelial dysfunction is present in brain dead subjects. Pulm Circ. 2013;3:419-25.

21. Cornet AD, Groeneveld AB, Hofstra JJ, et al. Recombinant human activated protein $C$ in the treatment of acute respiratory distress syndrome: a randomized clinical trial. PLoS ONE. 2014;9:e90983.

22. Langleben D, Orfanos SE, Giovinazzo M, et al. Pulmonary capillary endothelial metabolic dysfunction: severity in pulmonary arterial hypertension related to connective tissue disease versus idiopathic pulmonary arterial hypertension. Arthritis Rheum. 2008;58:1156-64.

23. Harel F, Langleben D, Provencher S, et al. Molecular imaging of the human pulmonary vascular endothelium in pulmonary hypertension: a phase II safety and proof of principle trial. Eur J Nucl Med Mol Imaging. 2017;44:1136-44. 